\title{
Amadurecimento precoce da placenta avaliada pela ultra-sonografia e prognóstico perinatal
}

\author{
Early placental maturation evaluated by ultrasound and perinatal prognosis
}

Ana Patrícia Santos de Queiroz ${ }^{1}$, Cícero Ferreira Fernandes Costa ${ }^{2}$

RESUM0

\begin{abstract}
Objetivo: descrever os resultados maternos e perinatais das gestações com amadurecimento precoce da placenta detectado pela ultra-sonografia. Métodos: realizou-se estudo retrospectivo, descritivo, tipo série de casos, com comparação de grupos. Foram incluídas 146 gestantes, internadas entre janeiro de 2000 e dezembro de 2002 e em cujo prontuário constavam diagnóstico de amadurecimento precoce da placenta (presença de placenta grau II antes da $32^{\mathrm{a}}$ semana de gestação ou grau III, antes da $35^{\text {a }}$ semana de gestação) e descrição das condições materno-fetais. Foram excluídas gestantes com diagnóstico de: amniorrexe prematura, gravidez múltipla, forma aguda de descolamento prematuro de placenta normoinserida e malformação fetal. As complicações clínico-obstétricas foram: doenças hipertensivas, redução do crescimento intra-uterino, alterações do volume de líquido amniótico, infecções, diabete materno, anemia falciforme, soropositividade para HIV, drogadição, litíase renal, epilepsia e asma brônquica. Pelos prontuários, foram identificadas 106 gestantes com complicações clínicoobstétricas (Gcom) e 40, sem essas complicações (Gsem). Para comparação entre os grupos, empregaram-se os testes de $\chi^{2}$ e exato de Fisher, ao nível de significância de 0,05 . Resultados: o grupo Gcom apresentou maior freqüência de oligoâmnio $(27,3 \%)$, restrição de crescimento intra-uterino $(44,3 \%)$ e cesárea antecedente ao trabalho de parto $(36,8 \%)$. Comparado ao grupo Gsem, o Gcom caracterizou-se por maior incidência de óbitos fetais, prematuros (58,8 versus $40 \%)$, menor índice de Apgar de $5^{\circ}$ minuto, peso ao nascer $<2.500 \mathrm{~g}(67,9$ versus $40 \%)$, recém-nascidos pequenos para a idade gestacional $(39,2$ versus 10\%) e intercorrências mais graves. Conclusões: o prognóstico perinatal não dependeu do amadurecimento precoce da placenta, mas sim da presença de complicações clínico-obstétricas maternas.
\end{abstract}

PALAVRAS-CHAVE: Placenta; Ultrasonografia pré-natal; Prognóstico

\section{ABSTRACT}

Purpose: to describe perinatal and obstetric characteristics of pregnant women with ultrasonographic early placental aging. Methods: using a retrospective, descriptive, series of cases, with group comparison, the authors analyzed the data of 146 pregnant women, whose diagnosis of placental early aging (presence of grade II placenta before 32 gestational weeks or grade III, before 35 gestational weeks), and maternal-fetal conditions had been recorded in the medical charts at the "Maternidade Prof. Monteiro de Moraes", Recife, Pernambuco - Brazil, from January 2000 to December 2002, where they had been attended as inpatients. The exclusion criteria were diagnoses of: premature amniorrhexis, multiple pregnancies, acute premature detachment of a normally located placenta, and fetal malformation. The clinical and obstetric complications were: hypertensive diseases, intrauterine growth restriction, changes of amniotic fluid volume, infections, maternal diabetes, falciform anemia, HIV seropositivity, drug addiction, renal lithiasis, epilepsy and bronchial asthma. In the medical records, 106 pregnant women were identified as having clinical and obstetric complications (Gwith group) and 40 as not having any of these complications (Gwithout group). For group comparisons, $\chi^{2}$ and exact Fisher statistical tests were used, with significance level of 0.05 . Results: Gwith group was associated with higher incidence of oligoamnion (27.3\%), intrauterine growth restriction (44.3\%) and caesarean section prior to labor (36.8\%). Compared to Gwithout, the Gwith group was characterized by high incidence of: fetal death, prematurity ( $58.8 \%$ versus $40 \%)$, lower $5^{\text {th }}$ minute Apgar index, birth weight less than $2.500 \mathrm{~g}(67.9 \%$ versus $40 \%)$; small body size for gestational age (39.2\% versus $10 \%)$ and more severe intercurrents events. Conclusions: perinatal prognosis does not depend upon placental early aging, but on clinical and obstetric maternal complications.

KEYWORDS: Placenta; Ultrasonography, prenatal; Prognosis

Trabalho realizado na Maternidade Professor Monteiro de Moraes da Universidade de Pernambuco - UPE - Recife (PE), Brasil.

1 Médica Tocoginecologista da Maternidade Professor Monteiro de Moraes da Universidade de Pernambuco - UPE - Recife (PE), Brasil e do Serviço de Medicina Fetal do Instituto Materno-Infantil Professor Fernando Figueira - IMIP - Recife (PE), Brasil.

2 Médico Tocoginecologista da Maternidade Professor Monteiro de Moraes da Universidade de Pernambuco - UPE - Recife (PE), Brasil.

Correspondência: Ana Patrícia Santos de Queiroz

Rua Isaac Salazar, n 189 apto 1802 - Tamarineira - 52050-160 - Recife - PE - Telefone: (81)-3304-4301 - e-mail: anapsqueiroz@uol.com.br 


\section{Introdução}

Em 1979, foi descrito método ultrasonográfico de classificação e graduação da maturidade placentária, que se associava à relação lecitina/esfingomielina, por meio da qual se evidencia a maturidade pulmonar fetal ${ }^{1}$. A classificação placentária nos graus 0, I, II e III baseia-se nas mudanças que ocorrem na placa coriônica, no tecido placentário e na lâmina basal ${ }^{1}$, compreendendo quatro fases progressivas e relativamente distintas de sua maturação, que se sucedem ao longo do processo gestacional.

As variações texturais podem corresponder a processos normais, mas devem ser diferenciadas dos processos patológicos. Embora algumas alterações placentárias estejam significantemente associadas com aumento da morbidade perinatal, a ocorrência de achados como sonolucências ou calcificações, isoladas ou associadas, não é indicativa do comprometimento do bem-estar fetal ${ }^{2}$.

A associação entre calcificação placentária e idade gestacional tem sido observada desde os primeiros estudos ultra-sonográficos sobre a placenta $^{1,3}$. Fato importante consiste na relação entre a calcificação placentária precoce (grau II e grau III placentário anterior à $32^{\mathrm{a}}$ e $35^{\mathrm{a}}$ semana de gestação, respectivamente) e a possibilidade de desenvolvimento de restrição de crescimento intra-uterino $(\mathrm{RCIU})^{4-6}$, causa freqüente de morbidade e mortalidade perinatal ${ }^{2}$.

Quando se observou ocorrência mais comum de placenta grau III prematura em gestantes hipertensas e com RCIU, foi sugerido que o aparecimento precoce de sonolucências e de calcificações na placenta pode representar disfunção ou senescência placentária e não maturação fisiológica $^{7}$. Posteriormente foi relatada associação entre placenta grau III com fetos pré-termo e complicações perinatais, em $78 \%$ dos casos, principalmente relacionadas com hipertensão induzida pela gestação, RCIU, descolamento prematuro da placenta e sofrimento fetal intraparto ${ }^{8}$.

A partir da conceituação de maturação placentária precoce, afastou-se seu papel como bom indicador para avaliação de prognóstico perinatal adverso ${ }^{9}$. Todavia, há maior possibilidade de complicações na gestação quando o amadurecimento placentário é precoce ${ }^{4,10-14}$, evento que se associa a maior incidência de sofrimento fetal, presença de líquido amniótico meconial, baixo índice de Apgar, baixo peso ao nascer e óbito perinatal.

Alguns estudos não revelam associação entre maturação placentária precoce e complicações na gravidez, nem identificam evidências de que as calcificações da placenta tenham algum significado clínico ou patológico ${ }^{15-17}$. No entanto, por meio de estudo controlado, foi observado que o acompanhamento ultra-sonográfico, com avaliação de maturidade placentária, volume de líquido amniótico e estimativa do peso fetal, serve como indicador do risco fetal em população de baixo ris$\mathrm{Co}^{18}$, principalmente na presença de grau III placentário antes da $36^{\mathrm{a}}$ semana de gestação, quando pode ajudar no diagnóstico de RCIU e na predição de subseqüente desenvolvimento de hipertensão induzida pela gestação ${ }^{19}$.

Existem controvérsias quanto à associação da precocidade de maturação placentária com complicações clínico-obstétricas maternas ou fetais. A presença dessa precocidade pode requerer acompanhamento mais intensivo da gestação, aumentando os custos com a assistência pré-natal, visto que se torna mais freqüente o uso de propedêutica complementar, ou mesmo pode determinar a interrupção iatrogênica da gravidez. Este trabalho poderá favorecer a obtenção de mais informações quanto à associação deste achado com o prognóstico perinatal e a maturidade pulmonar fetal, permitindo melhor abordagem e conduta nesses casos.

O objetivo do presente artigo foi, portanto, descrever os resultados maternos e perinatais das gestações nas quais se diagnosticou amadurecimento precoce da placenta.

\section{Métodos}

Realizou-se estudo descritivo, retrospectivo, tipo série de casos com comparação de grupos.

Foram incluídas as gestantes, internadas na enfermaria de gestação de alto risco e que pariram na Maternidade Prof. Monteiro de Moraes, Recife, Pernambuco, no período de janeiro de 2000 a dezembro de 2002, em cujo prontuário constavam diagnóstico ultra-sonográfico de amadurecimento precoce da placenta, firmado nessa Maternidade, e descrição das condições materno-fetais. Foram excluídas as pacientes com diagnósticos de: amniorrexe prematura, gravidez múltipla, forma aguda de descolamento prematuro da placenta normoinserida (DPPNI) e malformação fetal, associados ao de amadurecimento precoce de placenta.

A partir das estatísticas da Maternidade, admitindo prevalência de complicações perinatais igual a $78 \% \%^{8}$, determinou-se tamanho amostral igual a 150 gestantes empregando a fórmula de Milton e Tsokos, com precisão igual a 6,5\%, ao nivel de significância de 0,05. 
Pelo fato de ser maternidade-escola, há rotina estabelecida para, durante a internação na enfermaria de alto risco, submeter todas as gestantes à avaliação ultra-sonográfica. Foram localizadas, no arquivo do setor de ultra-sonografia e gestação de alto risco, anotações sobre 146 gestantes com amadurecimento precoce de placenta firmado no período de janeiro de 2000 a dezembro de 2002. Consultando os prontuários dessas gestantes, no Serviço de Arquivo Médico e Estatística da instituição, foram obtidas as demais informações necessárias à pesquisa, as quais foram registradas em ficha elaborada para coleta dos dados.

Classificou-se o amadurecimento placentário segundo os critérios de Grannum et al. ${ }^{1}$. A placenta grau 0 (G0) é nitidamente delineada, com a placa coriônica relativamente linear e textura homogênea. A placenta grau I (GI) é caracterizada por placa coriônica ligeiramente ondulada, com áreas ecogênicas dispersas. A placenta grau II (GII) é identificada pela presença de pequenas áreas ecogênicas ao longo da lâmina basal placentária e de divisões do tecido placentário por densidades ecogênicas em forma de vírgula, originadas da placa coriônica. A placenta grau III (GIII) é caracterizada por indentações ecogênicas que se estendem da placa coriônica à lâmina basal, dividindo a placenta em pequenos componentes (cotilédones).

Classificou-se o amadurecimento placentário como precoce na presença de placenta grau II antes da $32^{a}$ semana de gestação ou grau III quando antes de $35^{\mathrm{a}}$ semana de gestação.

Foram consideradas complicações clínicoobstétricas diagnosticadas no momento da ultrasonografia que caracterizou amadurecimento precoce da placenta: doenças hipertensivas (préeclâmpsia, hipertensão arterial crônica e hipertensão induzida pela gravidez), RCIU (peso ao nascer menor que o percentil 10), alterações do volume de líquido amniótico (oligoâmnio e polidrâmnio), infecções, diabete, anemia falciforme, soropositividade para HIV, abuso de droga, litiase renal, epilepsia e asma brônquica. A partir dos registros dos prontuários, identificaram-se 106 $(72,6 \%)$ gestantes com complicações clínico-obstétricas e $40(27,4 \%)$ gestantes sem tais complicações, que constituíram dois grupos, Gcom e Gsem, respectivamente.

Do concepto, foram analisados: peso ao nascer, índice de Apgar de $1^{\circ}$ e $5^{\circ}$ minuto, idade gestacional avaliada pelo método de Capurro e adequação do peso do RN à idade gestacional. As intercorrências perinatais precoces intra-hospitalares compreenderam: síndrome hipóxicoisquêmica (presença de dispnéia, cianose e estridor ou cornagem), doença da membrana hialina, taquipnéia transitória do recém-nascido, icterícia, óbito fetal intra-uterino e óbito neonatal precoce. As características maternas analisadas foram: idade, paridade, idade gestacional determinada no momento do diagnóstico de amadurecimento precoce de placenta e tipo de parto (normal, com ou sem uso de fórcipe e cesárea antecedente ao trabalho de parto ou intraparto).

Os dados foram organizados por meio do programa Epi-Info 2002, versão 1.0. Na descrição amostral, utilizaram-se: distribuição de freqüências absolutas e relativas, medidas de posição central e de dispersão. Para comparação dos dois grupos de gestantes, utilizaram-se os testes de $\chi^{2}$ e exato de Fisher, ambos com nível de significância de 0,05 .

O presente estudo atende à Resolução 196/ 1996 do Conselho Nacional de Saúde e às recomendações da Declaração de Helsinki VI, para pesquisa em seres humanos, tendo sido submetido e aprovado pela Comissão de Ética em Pesquisa do Centro Integrado à Saúde Amaury de Medeiros.

\section{Resultados}

A média de idade do grupo geral das gestan-

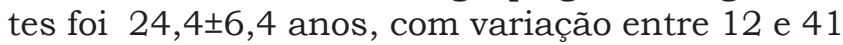
anos. Quanto à paridade, $77(52,8 \%)$ eram nuliparas, $38(26 \%)$ primiparas e $31(21,2 \%)$ multíparas.

No Gcom, as complicações clínico-obstétricas associadas ao amadurecimento precoce de placenta foram: doenças hipertensivas $(55,7 \%)$, restrição de crescimento intra-uterino $(44,3 \%)$, alterações do volume do líquido amniótico $(32,1 \%)$, das quais $85,3 \%$ era oligoâmnio e $14,7 \%$ polidrâmnio, diabete gestacional $(8,5 \%)$ e infecções $(0,9 \%)$. Outras doenças incluíram anemia falciforme, soropositividade de HIV, abuso de droga, litíase renal, epilepsia e asma brônquica. Dentre as 34 gestantes que apresentaram alterações de volume do líquido amniótico, em 29 (85,3\%) o oligoâmnio associou-se à doença hipertensiva e RCIU.

Para 76 mulheres $(52,1 \%)$, o parto foi transpélvico, com ou sem uso de fórcipe, sendo mais freqüente dentre as parturientes do Gsem que do Gcom (72,5 versus 44,3\%, respectivamente). A cesárea intraparto predominou entre as pacientes do Gsem (10 casos, 25\%) e a cesárea antecedente ao trabalho de parto por indicação clinica, no Gcom (39 casos, 36,8\%). Essas diferenças foram significantes $\left(\chi^{2}=10,09 ; p=0,001\right)$. 
Quanto à condição de nascimento, observouse que os conceptos de mães do Gcom nasceram e se mantiveram significantemente mais deprimidos que aqueles de mães do Gsem. Vinte e oito conceptos de mães do Gcom $(26,4 \%)$ apresentaram Apgar de $1^{\circ}$ minuto menor que 7 , dos quais 12 $(42,8 \%)$ permaneceram com Apgar baixo ao quinto minuto (Tabela 1).

Tabela 1 - Distribuição dos 146 conceptos de mães com e sem complicações clínicoobstétricas, segundo índices de Apgar.

\begin{tabular}{|c|c|c|c|c|c|c|c|}
\hline \multirow{3}{*}{$\begin{array}{l}\text { Características } \\
\text { ao nascimento }\end{array}$} & \multicolumn{4}{|c|}{ Número de casos } & \multirow{2}{*}{\multicolumn{2}{|c|}{ Total }} & \multirow[t]{3}{*}{ Testes } \\
\hline & \multicolumn{2}{|c|}{ Gcom } & \multicolumn{2}{|c|}{ Gsem } & & & \\
\hline & $\mathrm{n}$ & $\%$ & $\mathrm{n}$ & $\%$ & $\mathrm{n}$ & $\%$ & \\
\hline \multicolumn{8}{|l|}{ Índice de Apgar* } \\
\hline $1^{\circ}$ minuto & & & & & & & $\chi^{2}=2,27$ \\
\hline$<7$ & 28 & 26,4 & 5 & 12,8 & 33 & 22,8 & $p=0,13$ \\
\hline$\geq 7$ & 78 & 73,6 & 34 & 87,2 & 112 & 77,2 & \\
\hline $5^{\circ}$ minuto & & & & & & & Fisher $=0,02$ \\
\hline$<7$ & 12 & 11,3 & - & - & 12 & 8,3 & \\
\hline$\geq 7$ & 94 & 88,7 & & 100,0 & 133 & 91,7 & \\
\hline
\end{tabular}

Nota: Gcom - mães com complicações clínico-obstétricas, Gsem - mães sem complicações clínico-obstétricas.

${ }^{*} \mathrm{O}$ índice de Apgar de $1^{\circ}$ e de $5^{\circ}$ minuto de um recém-nascido foi omitido no prontuário neonatal.

A média de peso ao nascer dos conceptos de mães do Gcom foi $2.150 \pm 702 \mathrm{~g}$ e variou entre 650 e $3.800 \mathrm{~g}$, ao passo que no Gsem foi $2.633 \pm 659 \mathrm{~g}$, atingindo os limites de 1.210 a $4.100 \mathrm{~g}$, sendo a diferença significante. Houve maior número de conceptos com peso ao nascer inferior a $2.500 \mathrm{~g}$ no Gcom que no Gsem, com percentuais respectivamente iguais a 67,9 e $40 \%$, diferença significante (Tabela 2).

Tabela 2 - Distribuição dos 146 conceptos de mães com e sem complicações clínicoobstétricas, segundo peso ao nascer.

\begin{tabular}{lccccccc}
\hline $\begin{array}{l}\text { Características } \\
\text { ao nascimento }\end{array}$ & \multicolumn{2}{c}{$\begin{array}{c}\text { Número de casos } \\
\text { Gcom }\end{array}$} & \multicolumn{2}{c}{ Tsem } & & & Total \\
& $\mathbf{n}$ & $\%$ & $\mathbf{n}$ & $\%$ & $\mathbf{n}$ & $\%$ & \\
\hline Peso ao nascer $(\mathrm{g})$ & & & & & & & \\
$<1.500$ & 15 & 14,2 & 2 & 5,0 & 17 & 11,6 & $\chi^{2}=11,14$ \\
$1.500-1.999$ & 31 & 29,2 & 5 & 12,5 & 36 & 24,7 & $p=0,01$ \\
$2.000-2.499$ & 26 & 24,5 & 9 & 22,5 & 35 & 24,0 & \\
$\geq 2.500$ & 34 & 32,1 & 24 & 60,0 & 58 & 39,7 & \\
\hline
\end{tabular}

Nota: Gcom - mães com complicações clínico-obstétricas, Gsem - mães sem complicações clínico-obstétricas.

Comparando a distribuição de recém-nascidos por peso, segundo a idade gestacional nos dois grupos, identificou-se que a ocorrência de pequeno para a idade gestacional (PIG) foi significante- mente maior no Gcom (39,2 versus 10\% no Gsem). Sessenta $(58,8 \%)$ conceptos de mães com complicações clínico-obstétricas nasceram com menos de 37 semanas de gestação, avaliadas pelo método de Capurro. No Gsem, esse percentual igualouse a $40 \%$, diferença estatisticamente significante (Tabela 3).

Tabela 3 - Distribuição dos 146 conceptos de mães com e sem complicações clínicoobstétricas, segundo adequação do peso à idade gestacional e idade gestacional pelo método de Capurro.

\begin{tabular}{|c|c|c|c|c|c|c|c|}
\hline \multirow{3}{*}{$\begin{array}{l}\text { Características } \\
\text { ao nascimento }\end{array}$} & \multicolumn{4}{|c|}{ Número de casos } & \multirow{2}{*}{\multicolumn{2}{|c|}{ Total }} & \multirow[t]{3}{*}{ Testes } \\
\hline & \multicolumn{2}{|c|}{ Gcom } & \multicolumn{2}{|c|}{ Gsem } & & & \\
\hline & $n$ & $\%$ & $n$ & $\%$ & $n$ & $\%$ & \\
\hline $\begin{array}{l}\text { Adequaçã̃o do peso à } \\
\text { idade gestacional" }\end{array}$ & & & & & & & $\chi^{2}=12,84$ \\
\hline adequado (AIG) & 61 & 59,8 & 34 & 85,0 & & 566,9 & $p=0,002$ \\
\hline pequeno (PIG) & 40 & 39,2 & 4 & 10,0 & & 431,0 & \\
\hline grande (GIG) & 1 & 1,0 & 2 & 5,0 & & 32,1 & \\
\hline $\begin{array}{l}\text { Idade gestacional pelo } \\
\text { método de Capurro } \\
\text { (semanas gestacionais) }\end{array}$ & & & & & & & $\chi^{2}=4,09$ \\
\hline$<37$ & 60 & 58,8 & 16 & 40 & & 653,5 & $p=0,04$ \\
\hline$\geq 37$ & 42 & 41,2 & 24 & 60 & & 646,5 & \\
\hline
\end{tabular}

Nota: Gcom - mães com complicações clínico-obstétricas, Gsem - mães sem complicações clínico-obstétricas.

"Adequação à idade gestacional - foram excluídos os quatro óbitos fetais no Gcom.

Foram verificados quatro $(57,1 \%)$ óbitos fetais, todos no Gcom, e três (42,9\%) óbitos neonatais precoces intra-hospitalares, dos quais um $(33,3 \%)$ ocorreu no Gsem. Dentre os 142 neonatos vivos, foram diagnosticadas intercorrências neonatais em $64(45,1 \%)$ do Gcom e em 21 $(14,8 \%)$ do Gsem. As intercorrências mais graves (síndrome hipóxico-isquêmica e doença da membrana hialina) foram significantemente mais freqüentes no Gcom (51,5\% dos conceptos versus $42,8 \%$ do Gsem).

\section{Discussão}

No Gcom, as complicações clínico-obstétricas foram as doenças hipertensivas, a RCIU e o oligoâmnio, concordando com os achados de outros autores ${ }^{4,7,8,10}$. Essa associação talvez possa ser justificada pela própria fisiopatologia da préeclâmpsia, na qual há déficit no fluxo útero-placentário, levando à insuficiência placentária e conseqüentes oligoâmnio e RCIU.

No Gcom, apesar de $44,3 \%$ dos fetos terem sido considerados portadores de RCIU, esse diagnóstico foi menos freqüente quando comparado a 
outros estudos ${ }^{4,7-10}$. Nas gestantes do Gsem, o amadurecimento precoce de placenta isolado não determinou aumento na incidência de RCIU, dado semelhante ao de outros estudos ${ }^{15,16}$ para avaliação de gestação de baixo risco.

Por se tratar de maternidade-escola, na qual as gestações de alto risco são sistematicamente discutidas em reuniões clínicas, o número de cesáreas antecedendo o trabalho de parto foi mais freqüente no Gcom em decorrência de RCIU, préeclâmpsia grave e oligoâmnio. $\mathrm{O}$ achado isolado de amadurecimento precoce de placenta não justifica a conduta ativa quanto à interrupção da gestação, mas, sim, o contexto clínico no qual ele está inserido.

O predomínio de parto normal no Gsem derivou da admissão das pacientes em trabalho de parto prematuro espontâneo, o qual não foi inibido devido à presença de placenta grau III ou à fase avançada da parturição, dispensando assim indicar útero-inibição. Além disso, os fetos apresentavam-se hígidos, não havendo indicação para cesárea.

O grau III placentário está associado a fetos com baixos índices de Apgar ao nascimento ${ }^{13}$, no entanto o achado de maturação placentária precoce tem baixa sensibilidade em predizer resultados perinatais adversos ${ }^{9}$. Analisando as condições de nascimento dos recém-nascidos pelo índice de Apgar, os conceptos do Gcom se mostravam ainda deprimidos ao quinto minuto, contrariamente ao que ocorreu naqueles do Gsem. Talvez esse fato se deva não apenas à presença de complicações clínico-obstétricas, mas também à relação existente entre maturação placentária e maturidade pulmonar fetal. Em pacientes próximas do termo, sem complicações maternas associadas à gestação, a presença de placenta grau III é indicador forte de maturidade pulmonar fetal, todavia, na presença de hipertensão arterial crônica, a maturidade placentária pode anteceder a pulmonar fetal ${ }^{10}$.

A associação de amadurecimento precoce da placenta e baixo peso ao nascer reflete aumento no risco de parto prematuro e de recém-nascidos $\mathrm{PIG}^{11}$. Essa associação é ainda mais importante na presença de complicações clínico-obstétricas, o que explica a incidência significantemente maior de fetos com baixo peso ao nascer e PIG no Gcom.

O grau III placentário também se associa a óbito perinatal $2,4-8,11$. Esse achado foi observado em sete $(4,8 \%)$ casos, seis $(85,7 \%)$ dos quais em gestações complicadas com pré-eclâmpsia, oligoâmnio e RCIU, e um (14,3\%), em ausência de complicações clínico-obstétricas. Assim, nos casos com complicações clínico-obstétricas, o óbito perinatal pode ter relação com insuficiência placentária, ao passo que, nas gestantes sem essas complicações, a prematuridade pode ter atuado como principal fator determinante de tais óbitos.

Em ambos os grupos, a taxa de mortalidade neonatal foi elevada. No Gcom, ocorreram todos os óbitos fetais, ao passo que no Gsem, o óbito foi neonatal intra-hospitalar, o que permite aventar a hipótese da contribuição do amadurecimento precoce de placenta, associado às complicações clínico-obstétricas, na precocidade desses óbitos do Gcom.

Em ambos os grupos, a intercorrência neonatal precoce intra-hospitalar mais comum foi a taquipnéia transitória, seguida da doença da membrana hialina. Apresentaram doença da membrana hialina 32,3\% dos recém-nascidos de mães do Gcom e $25 \%$ daqueles de mães do Gsem, em que, na maioria dos casos, manifestou-se na forma leve, tendo os recém-nascidos sobrevivido. Talvez o fato de esses conceptos terem nascido em hospital de referência, além de terem recebido assistência neonatal imediata, possa explicar os resultados obtidos. A literatura refere que todos os casos de doença da membrana hialina estiveram associados à placenta grau III em mães com hipertensão arterial crônica e com parto prematu$\mathrm{ro}^{10}$. Isso sugere a hipótese de que, em pacientes com hipertensão arterial crônica, as mudanças na textura placentária são aceleradas, com o surgimento de calcificações, antes de ocorrer maturidade pulmonar fetal.

Desse modo, diante do exposto, evidencia-se a necessidade de acompanhamento clínico obstétrico mais intensivo nos casos de diagnóstico de amadurecimento precoce da placenta com exames complementares, sendo a dopplervelocimetria materno-fetal fundamental, principalmente nas gestantes com complicações clínico-obstétricas.

Conclui-se que o amadurecimento precoce de placenta associado a complicações clínico-obstétricas representa risco de piores resultados perinatais, embora isoladamente não determine pior prognóstico ao nascimento.

Os autores declaram não haver conflito de interesses.

\section{Referências}

1. Grannum PA, Berkowitz RL, Hobbins JC. The ultrasonic changes in the maturing placenta and their relation to fetal pulmonic maturity. Am J Obstet Gynecol. 1979;133(8):915-22. 
2. Kuhlmann RS, Warsof S. Ultrasound of the placenta. Clin Obstet Gynecol. 1996;39(3):519-34.

3. Zanforlin Filho SM. Placenta. In: Pastore AR, Cerri GG, editores. Ultra-sonografia em obstetricia e ginecologia. São Paulo: Sarvier; 1996. p. 139-57.

4. Kazzi GM, Gross TL, Sokol RJ, Kazzi NJ. Detection of intrauterine growth retardation: a new use for sonographic placental grading. Am J Obstet Gynecol. 1983;145(6):733-7.

5. Resnik R. Intrauterine growth restriction. Obstet Gynecol. 2002;99(3):490-6.

6. Regnault TR, Galan HL, Parker TA, Anthony RV. Placental development in normal and compromised pregnancies: a review. Placenta. 2002;23 Suppl A:S119-29.

7. Quinlan RW, Cruz AC, Buhi WC, Martin M. Changes in placental ultrasonic appearance I. Incidence of grade III changes in the placenta in correlation to fetal pulmonary maturity. Am J Obstet Gynecol. 1982;144(4):468-70.

8. Quinlan RW, Cruz AC, Buhi WC, Martin M. Changes in placental ultrasonic appearance II. Pathologic significance of grade III placental changes. Am J Obstet Gynecol. 1982;144(4):471-3.

9. Patterson RM, Hayashi RH, Cavazos D. Ultrasonographically observed early placental maturation and perinatal outcome. Am J Obstet Gynecol. 1983;147(7):773-7.

10. Kazzi GM, Gross TL, Rosen MG, Jaatoul-Kazzi NY. The relationship of placental grade, fetal lung maturity, and neonatal outcome in normal and complicated pregnancies. Am J Obstet Gynecol. 1984;148(1):54-8.
11. Proud J, Grant AM. Third trimester placental grading by ultrasonography as a test fetal well-being. Br Med J (Clin Res Ed). 1987;294(6588):1641-4.

12. Chitlange SM, Hazari KT, Joshi JV, Shah RK, Mehta AC. Ultrasonographically observed preterm grade III placenta and perinatal outcome. Int $\mathrm{J}$ Gynaecol Obstet. 1990;31(4):325-8.

13. Laske MC, Gonçalves LFA, Saab Neto, JA. Maturação placentária precoce. ACM Arq Catarin Med. 1998;27(1/4):39-44.

14. Baeza Valenzuela A, García Méndez A. Envejecimiento prematuro de placenta: diagnóstico por ultrasonido. Ginecol Obstet Mex. 1995;63(7):287-92.

15. Vosmar MB, Jongsma HW, van Dongen PW. The value of ultrasonic placental grading: no correlation with intrauterine growth retardation or with maternal smoking. J Perinat Med. 1989;17(2):137-43.

16. Montan S, Jörgensen C, Svalenius EM, Ingemarsson I. Placental grading with ultrasound in hypertensive and normotensive pregnancies. A prospective, consecutive study. Acta Obstet Gynecol Scand. 1986;65(5):477-80.

17. Suetake H. Implicações clínicas do diagnóstico ultra-sonográfico de placenta grau III [dissertação]. Botucatu: Universidade Estadual Paulista; 2001.

18. McKenna D, Tharmaratnam S, Mahsud S, Bailie C, Harper A, Dornan J. A randomized trial using ultrasound to identify the high-risk fetus in a lowrisk population. Obstet Gynecol. 2003;101(4):62632.

19. McKenna D, Tharmaratnam S, Mahsud S, Dornan J. Ultrasonic evidence of placental calcification at 36 weeks' gestation: maternal and fetal outocomes. Acta Obstet Gynecol Scand. 2005;84(1):7-10. 